\title{
El impacto de las remesas internacionales sobre la inversión en educación en la localidad de Caltimacán, Hidalgo
}

\section{The Impact of International Remittances on Investment in Education in Caltimacán, Hidalgo}

\author{
Yesenia García Nájera ${ }^{1}$ y Alfredo Cuecuecha Mendoza ${ }^{2}$ \\ RESUMEN
}

Este artículo presenta resultados de una investigación que analiza el impacto de las remesas internacionales sobre inversión en educación en Caltimacán, estado de Hidalgo, México, en una comunidad que se caracteriza por ser de alta migración basada en contratos temporales de trabajo. Para resolver los problemas de sesgo originados por la simultaneidad existente entre la recepción de las remesas y las inversiones en educación, así como por la existencia de variables no observadas que determinan a ambas variables, se utiliza el Método de las Variables Instrumentales, utilizando para dicho fin a las tasas de creación de empleo en los Estados de la Unión Americana en los que trabajaban los migrantes durante el periodo comprendido entre el 2010 y el 2011. Los resultados muestran que las remesas aumentan cuando hay una mayor creación de empleo en la Unión Americana, lo que a su vez genera una mayor inversión en educación en hogares receptores de remesas, en comparación con hogares no receptores. Sin embargo, este impacto desaparece al incluir en el modelo el gasto de los hogares y el nivel de riqueza de los mismos. Estos resultados confirman que la inversión en educación en Caltimacán se ve sujeta a restricciones de acceso a crédito, mismas que son suavizadas por la llegada de las remesas.

Palabras clave: 1. remesas, 2. inversión en educación, 3. restricciones crediticias. 4. Caltimacán 5. estado Hidalgo.

\begin{abstract}
This article presents the results of a study about the impact of international remittances on investment in education in the Mexican town of Caltimacán, Hidalgo, Mexico, a community that is characterized by legal migration through temporary labor contracts. The estimations apply instrumental variables based on job creation in the American states were migrants worked between 2010 and 2016. The results show that the proportion of expenditure spent on education is higher among households that receive remittances than among households that do not receive them. However, this effect vanishes when household expenditure and wealth are introduced in the model. These results confirm that the investments in the education of the inhabitants of Caltimacán face credit constraints, which in turn are diminish by the reception of remittances.
\end{abstract}

Keywords: 1. remittances, 2. education investment, 3. credit constraints, 4. Caltimacán, 5. Hidalgo.

Fecha de recepción: 19 de julio de 2017

Fecha de aceptación: 20 noviembre de 2018

Publicación en web: 3 de abril de 2020

${ }^{1}$ El Colegio de Tlaxcala, México, yesenia.garcianj@gmail.com,

${ }^{2}$ Centro de investigación e inteligencia económicas, UPAEP, México, alfredo.cuecuecha@upaep.mx, https://orcid.org/0000-0003-2828-0473 


\section{INTRODUCCIÓN}

En el año 2017, las remesas alcanzaron el nivel récord de 618000 millones de dólares de los Estados Unidos de América (World Bank Group, 2018). En México, en el mismo periodo, las remesas alcanzaron el record histórico de 28700 millones de dólares (Banco de México, 2018). Los efectos económicos y sociales a escala macroeconómica y microeconómica de estos extraordinarios flujos de efectivo han generado debates en la literatura (Adams y Cuecuecha, 2010, 2013; Buvinic y Gupta, 1997; Cuecuecha y Adams, 2016; Kandell y Massey, 2002; Levitt, 1998; Sawyer, 2015; Stark y Bloom, 1985). El presente trabajo tiene como objetivo ampliar el conocimiento que se tiene sobre la relación entre las remesas y el gasto en educación a nivel microeconómico para la localidad de Caltimacán, en el estado de Hidalgo, en México.

A nivel teórico, las remesas pueden tener tres tipos de efectos sobre la inversión en educación de los hogares:

1) ningún efecto, si las remesas son perfectamente fungibles y solamente sustituyen uno a uno los recursos que se perdieron en el hogar al perder miembros debido a la migración, y si la migración no genera cambios en la forma de operar del hogar;

2) un efecto positivo, que puede darse si las remesas son vistas como un recurso temporal que debe invertirse mientras dure;

3) un efecto negativo, si las remesas son vistas como un aumento de ingreso permanente, y el hogar decide aumentar su consumo y reducir sus niveles de inversión (Adams y Cuecuecha, 2010).

Las remesas también pueden tener un impacto positivo si los mercados de seguros y créditos son incompletos o faltantes, por lo que la estrategia de enviar migrantes y recibir remesas ayuda a concretar inversiones que el hogar no realizó debido a que no tuvo acceso a un crédito (Stark y Bloom, 1985). Las remesas también pueden tener impactos al cambiar la estructura del hogar, pues si los miembros del hogar que emigraron a los Estados Unidos y que envían las remesas son hombres, la jefatura del hogar puede volverse femenina y por ese hecho, aumentan las inversiones del hogar (Buvinic y Gupta, 1997). Finalmente, los hábitos de consumo también pueden modificarse en los hogares receptores de remesas si los miembros del hogar que viven en el extranjero influencian los hábitos de consumo cuando visitan a sus familiares en México (Levitt, 1998).

Estudios empíricos hechos para Guatemala (Adams y Cuecuecha, 2010), Ghana (Adams y Cuecuecha, 2013), Indonesia (Cuecuecha y Adams, 2016) y la comunidad de San Miguel Tlacotepec, en Oaxaca, México (Sawyer, 2015), reportan el impacto positivo de las remesas sobre el gasto de los hogares en educación. Kandell y Massey (2002) reportan resultados 
positivos y negativos, dependiendo de la muestra estudiada, por lo que recomiendan estudios a nivel de localidad para tratar de comprender mejor los casos específicos.

Debido a lo anterior, este trabajo se propone estudiar la localidad de Caltimacán, estado de Hidalgo, que se caracteriza por alta migración y alta recepción de remesas, así como porque sus migrantes tienen contratos temporales de trabajo en los Estados Unidos. Este estudio a nivel localidad permite medir con mayor precisión elementos que en otros estudios no se han podido incluir, tales como la aversión al riesgo y medidas de bienestar subjetivo. Para identificar el impacto de las remesas a nivel de hogar, se utiliza el hecho de que nuestra encuesta permite identificar el lugar de residencia en los Estados Unidos de los migrantes que envían remesas.

Esta información fue utilizada para construir un instrumento que mide la creación de empleo en el estado de residencia de los migrantes en Estados Unidos. El Método de las Variables Instrumentales permite encontrar el efecto causal de una variable, condicionando en un conjunto de variables observadas específicas de los hogares incluídos en la muestra (Wooldridge, 2013, p. 514).

El presente estudio muestra que un incremento en el empleo en Estados Unidos aumenta las remesas recibidas en México, lo que se traduce en un mayor gasto en educación. Lo anterior demostraría que las remesas son vistas como temporales, como ha sido reportado para otros países (Adams y Cuecuecha, 2010, 2013). A pesar de lo anterior, el efecto mencionado es fundamentalmente explicado por la mayor presencia de riqueza y de ingreso en dichos hogares.

Este resultado confirmaría que las remesas ayudan a realizar inversiones que el hogar tenía suspendidas por falta de acceso a crédito (Stark y Bloom, 1985), pues al introducir en el modelo dos factores que se asocian al acceso al crédito, tales como el ingreso y la riqueza (Ray, 1998, p. 268), el impacto positivo de las remesas desaparece. El resultado también implica que los hogares receptores de remesas gastan en educación de igual manera que los hogares no receptores de remesas con ingresos tan altos como los primeros. Es importante mencionar que debido a la representatividad de la muestra, el coeficiente estimado es válido para comunidades cuyos hogares tienen características similares a los de Caltimacán, Hidalgo (Deaton, 1997).

Existen otros estudios que han enfocado su atención en otras dimensiones de los indicadores de resultados asociados a la educación, tales como el abandono escolar (Cox Edwards y Ureta, 2003), la participación en la educación superior (Yang, 2008), la asistencia escolar (McKenzie y Rapoport, 2006; Hanson y Woodroff, 2003), el analfabetismo (López Cordova, 2005), las aspiraciones escolares (Pérez Gañán y Pesántez Calle, 2017) y la permanencia escolar (Acosta Rangel y Caamal Olvera, 2017). Cox Edwards y Ureta (2003) estiman las posibilidades del abandono escolar en El Salvador y encuentran que, a diferencia de las zonas rurales, en áreas urbanas las remesas disminuyen la deserción escolar. Yang 
(2008) estudia el caso de Filipinas y muestra que los jóvenes de 17 a 21 años de edad aumentan su participación en la educación superior. Pérez Gañán y Pesántez Calle (2017) señalan que cuando se reciben remesas, se mejoran las aspiraciones escolares de los jóvenes ecuatorianos.

Para el caso de México, McKenzie y Rapoport (2006) y Hanson y Woodroff (2003) encuentran que las remesas mejoran la asistencia escolar entre jóvenes de 13 a 15 años, particularmente de las niñas; por su parte, López Córdova (2005) encuentra que en los municipios mexicanos que reciben más remesas ha disminuido el analfabetismo en niños de entre 6 y 14 años. Recientemente, Acosta Rangel y Caamal Olvera (2017) analizan el efecto de las remesas sobre la permanencia escolar en México utilizando la técnica de paneles sintéticos, con datos de la ENIGH 2004 y 2008; en su estudio concluyen que mientras mayor sea la proporción de remesas, la permanencia escolar será más fuerte, ya que estos recursos adicionales permiten continuar invirtiendo en educación, sobre todo en hogares con alta marginación.

Otros trabajos han tratado de estudiar la relación entre la educación y las remesas de una manera indirecta, al estudiar el impacto de la migración sobre la educación de los hogares con migrantes en comparación a hogares sin migrantes. Algunos autores reportan una relación positiva (Hanson, Robertson y Spilimbergo, 2002; McKenzie y Rapoport, 2006), mientras que otros reportan una relación negativa (Bryant, Giorguli Saucedo, y Hernández Padilla, 2016).

El artículo se organiza de la siguiente manera: en la primera parte se describe la localidad de estudio, la encuesta realizada y los principales resultados de la encuesta; en la segunda parte se muestra el modelo empírico estimado en el estudio que expone los principales resultados; en la última parte se presentan las conclusiones del estudio.

\section{LOCALIDAD DE ESTUDIO, INSTRUMENTO UTILIZADO Y CARACTERIZACIÓN DE LOS HOGARES RECEPTORES DE REMESAS EN CALTIMACÁN, HIDALGO}

La comunidad de Caltimacán, en el estado de Hidalgo, pertenece al municipio de Tasquillo, considerado actualmente como de alta migración hacia Estados Unidos. La migración en esta región tiene antecedentes al programa Bracero, el cual ocurrió durante lo años 40 del siglo XX, beneficiando a todo México (Massey, 2016; Durand, 2016); la migración creció exponencialmente a principios de los años ochenta del siglo pasado.

De acuerdo a Quezada Ramírez (2008), al principio la población migrante de Caltimacán principalmente incluía a hombres con edades de entre 18 y 59 años, quienes laboraban en distintos estados de la Unión Americana, sobre todo en las actividades de construcción, jardinería y labores del campo. Típicamente, se trata de trabajos realizados entre los meses 
de febrero a noviembre, bajo contrato. A lo largo del tiempo, la población de origen caltimaqueño se ha esparcido a 17 estados de la Unión Americana, destacando en primer lugar los migrantes asentados en Texas, con un 45.63 por ciento; el segundo lugar, Indiana con 9.71 por ciento; el tercer lugar, Kansas con 5.83 por ciento; el cuarto lugar, California con 4.85 por ciento: el quinto lugar los ocupan las entidades de Carolina del Norte, Florida, Tennessee y Virginia, con un 3.88 por ciento, mientras que el resto de migrantes caltimeños se distribuye, en orden de importancia, en Arizona, Chicago, Misuri, Pensilvania, Arkansas, Dakota del Norte, Maryland, Oklahoma y Utah.

Quezada Ramírez (2008) argumenta que la migración impactó positivamente la economía local debido a un incremento en la construcción de casas y, en general, ha generado que la localidad de Caltimacán, Estado de Hidalgo, se encuentre con un grado de marginación moderado y por debajo de la media estatal y nacional (Quezada Ramírez, 2008). García Nájera (2017) muestra que las remesas internacionales tienen diferentes efectos en los hogares, principalmente en el bienestar subjetivo de las familias de la localidad estudiada.

En este estudio se utiliza la Encuesta de Recepción de Remesas Internacionales en Caltimacán, Hidalgo (ERRICH), que obtiene información acerca de la migración, las remesas y otras características sociales, económicas y demográficas de los hogares de dicha población (García Nájera, 2017). El tamaño de muestra de la ERRICH se calculó usando la metodología propuesta para proporciones por Valdivieso Taborga, Valdivieso Castellón y Valdivieso Taborga (2011), que estima el número de observaciones necesario para tener una muestra representativa. Es necesario realizar la encuesta buscando que sea representativa de la población que recibe las remesas, contextualizado la localidad estudiada. Para entender este argumento, basta decir que en 2017, a nivel nacional 4.8 por ciento de los hogares recibía remesas (Fundación BBVA, 2017), mientras que en la localidad de Caltimacán, dicha proporción para el año 2010 era de 92 por ciento, aproximadamente (Conapo, 2010). Dado un intervalo de confianza de 95 por ciento y un error de muestreo de 10 por ciento, se tiene que el tamaño de muestra es de 102 viviendas.

El cuestionario recopiló información relativa a la existencia de familiares viviendo en los Estados Unidos, y sobre aspectos específicos de la recepción de remesas; específicamente se preguntó: a) tipo de migrante en la vivienda (migrante reciente, migrante circular o migrante de retorno definitivo), b) si el migrante era el jefe del hogar, c) número de migrantes en el hogar, d) frecuencia de recepción de remesas (semanal, quincenal, mensual y bimestral), y e) monto de las remesas recibidas (menos de 100 dólares a más de 200 dólares).

En el caso de migrante reciente, se define como tal a aquellas personas con migración realizada 5 años antes de la encuesta (Inegi, 
2010), para el caso de migrante circular, este se define como a los migrantes que de alguna forma legal logran ir y venir entre los dos países (Alaminos, López Fernández, López Monzalve, Perea Crespo y Santacreu, 2009), y para el caso de migrante de retorno definitivo, se trata de aquellos que tuvieron experiencia migrante internacional $y$ que han regresado a México de forma definitiva.

El cuestionario también recogió información sobre los gastos en la familia, el cual concentra los gastos en el hogar en siete rubros: a) alimentación (artículos alimentarios), b) salud (medicamentos y tratamientos), c) colegiaturas (colegiaturas), d) otros gastos relacionados con la educación (materiales didácticos y transporte para ir a la escuela), e) esparcimiento y diversión, f) mantenimiento de la vivienda (construcción, jardinería, plomería y otros) y g) ayuda a un familiar; todos estos gastos fueron proporcionados como el gasto mensual en moneda nacional.

Este instrumento captó información no solo de los hogares que reciben remesas internacionales, sino también de aquellos que no cuentan con este ingreso; cabe aclarar que los datos recopilados para este estudio fueron proporcionados por la persona que se encontrara disponible al momento de la encuesta, aunque se procuró que fuera la esposa del jefe del hogar quien contestara la encuesta. Son datos de corte transversal levantados entre el 30 de mayo de 2016 y el 28 de julio de 2016.

El Cuadro 1 muestra información estadística sobre los hogares de Caltimacán, presentando algunas características de los hogares y clasificándolos de acuerdo a la recepción de remesas. La recepción de remesas internacionales en Caltimacán es importante ya que, del total de hogares, 81.6 por ciento recibe este recurso económico.

Los hogares de Caltimacán tienen un gasto promedio mensual de 8300 pesos mexicanos; los hogares con remesas tienen un gasto de 9218 pesos, y los hogares sin remesas tienen un gasto de 4291 pesos; la diferencia entre ambas variables es significativa al 1 por ciento. El gasto promedio en educación en Caltimacán es de 1911 pesos mensuales; los hogares con remesas gastan 2239 pesos mensuales, mientras que los hogares sin remesas gastan 480 pesos mensuales; la diferencia es significativa al 5 por ciento. El Cuadro 1 muestra que los hogares gastan 17 por ciento de su gasto en educación. Los hogares que reciben remesas tienen una proporción de gastos en educación de 19 por ciento; mientras que los hogares que no reciben remesas tienen una proporción de gasto en educación de 8 por ciento. Esta diferencia es significativa al 5 por ciento. Es importante mencionar que estas diferencias solo representan una asociación positiva entre los gastos en educación y las remesas, pues para determinar un efecto causal es necesario controlar por variables observadas, así como por otros posibles sesgos que existen al estudiar las remesas (Adams, 2011).

Cuadro 1. Estadística descriptiva, Caltimacán, Hidalgo, 2016 
\begin{tabular}{r|l} 
MIGRACIONES INTERNACIONALES, VOL. 11, ART. 5, 2020 & 7 \\
e-ISSN 2594-0279 http://dx.doi.org/10.33679/rmi.v1i1.1590 & r.
\end{tabular}

\begin{tabular}{|c|c|c|c|c|}
\hline Variable & $\begin{array}{c}\text { Total de } \\
\text { hogares en } \\
\text { Caltimacán }\end{array}$ & $\begin{array}{l}\text { Hogares } \\
\text { que reciben } \\
\text { remesas } \\
(1)\end{array}$ & $\begin{array}{l}\text { Hogares } \\
\text { que no } \\
\text { reciben } \\
\text { remesas } \\
\text { (2) }\end{array}$ & $\begin{array}{c}\text { Nivel de } \\
\text { significancia } \\
\text { de la } \\
\text { diferencia (1) } \\
\text { y (2) }\end{array}$ \\
\hline$\%$ Remesas en el hogar & 81 & 100 & 0 & NA \\
\hline Gasto mensual total en el hogar & $\begin{array}{c}8300.04 \\
{[5353.29]}\end{array}$ & $\begin{array}{c}9218 \\
{[5485]}\end{array}$ & $\begin{array}{c}4291 \\
{[1699]}\end{array}$ & $1 \%$ \\
\hline Gasto mensual en educación & $\begin{array}{c}1911.30 \\
{[3067.17]}\end{array}$ & $\begin{array}{c}2239 \\
{[3271]}\end{array}$ & $\begin{array}{c}480 \\
{[1181]}\end{array}$ & $5 \%$ \\
\hline$\%$ de gasto en educación & $\begin{array}{c}17 \\
{[0.21]}\end{array}$ & $\begin{array}{c}19 \\
{[0.21]}\end{array}$ & $\begin{array}{c}8 \\
{[0.2]}\end{array}$ & $5 \%$ \\
\hline Índice de no carencias & $\begin{array}{c}0.92 \\
{[0.11]}\end{array}$ & $\begin{array}{c}0.94 \\
{[0.01]}\end{array}$ & $\begin{array}{c}0.81 \\
{[0.08]}\end{array}$ & $1 \%$ \\
\hline Aversión al riesgo & $\begin{array}{c}0.47 \\
{[0.50]}\end{array}$ & $\begin{array}{c}0.43 \\
{[0.50]}\end{array}$ & $\begin{array}{c}0.63 \\
{[0.50]}\end{array}$ & NS \\
\hline Satisfacción ante la vida & $\begin{array}{c}0.62 \\
{[0.49]}\end{array}$ & $\begin{array}{c}0.64 \\
{[0.48]}\end{array}$ & $\begin{array}{c}0.53 \\
{[0.51]}\end{array}$ & NS \\
\hline Integrantes & $\begin{array}{c}3.88 \\
{[1.26]}\end{array}$ & $\begin{array}{c}3.89 \\
{[1.20]}\end{array}$ & $\begin{array}{c}3.84 \\
{[1.54]}\end{array}$ & NS \\
\hline$\%$ Menores de 5 años & $\begin{array}{c}20 \\
{[0.40]}\end{array}$ & $\begin{array}{c}14 \\
{[0.35]}\end{array}$ & $\begin{array}{c}42 \\
{[0.51]}\end{array}$ & $1 \%$ \\
\hline$\%$ Niños mayores de 5 años & $\begin{array}{c}43 \\
{[0.50]}\end{array}$ & $\begin{array}{c}47 \\
{[0.50]}\end{array}$ & $\begin{array}{c}26 \\
{[0.45]}\end{array}$ & NS \\
\hline Edad jefe & $\begin{array}{c}50.42 \\
{[17.54]}\end{array}$ & $\begin{array}{c}50.78 \\
{[16.54]}\end{array}$ & $\begin{array}{c}48.84 \\
{[21.83]}\end{array}$ & NS \\
\hline Edad madre & $\begin{array}{c}38.25 \\
{[20.34]}\end{array}$ & $\begin{array}{c}39.80 \\
{[19.59]}\end{array}$ & $\begin{array}{c}31.53 \\
{[22.67]}\end{array}$ & NS \\
\hline Educación jefe & $\begin{array}{c}8.08 \\
{[3.50]}\end{array}$ & $\begin{array}{c}7.89 \\
{[3.58]}\end{array}$ & $\begin{array}{c}8.89 \\
{[3.07]}\end{array}$ & NS \\
\hline Educación madre & $\begin{array}{c}7.91 \\
{[4.96]}\end{array}$ & $\begin{array}{c}8.10 \\
{[4.95]}\end{array}$ & $\begin{array}{c}7.11 \\
{[5.09]}\end{array}$ & NS \\
\hline $\mathrm{N}$ & 102 & 83 & 19 & \\
\hline
\end{tabular}

Notas: N=102 hogares. Todos los valores son ponderados; errores estándar en paréntesis.

El gasto total en el hogar y el gasto en educación esta medido en pesos mexicanos.

NA: no aplica. NS: no significativa.

Fuente: Elaboración propia con base en datos de la ERRICH-2016.

El Cuadro 1 presenta otras características de los hogares de Caltimacán, aunque no todas ellas pueden ser utilizadas en el análisis estadístico debido al número de observaciones; sin embargo, se presentan descriptivamente para lograr dar una mejor caracterización de los hogares receptores de remesas en Caltimacán, Hidalgo.

El índice de no carencias, que es un indicador de la riqueza de las familias y, por lo tanto, del bienestar objetivo de las mismas, marca que en promedio las familias de Caltimacán 
tienen un índice de 0.92 unidades; los hogares que reciben remesas tienen un índice de 0.94 unidades; mientras que los hogares sin remesas tienen un índice de 0.81 unidades; la diferencia es estadísticamente significativa al 1 por ciento. Es importante destacar que este resultado nos muestra que en Caltimacán los hogares receptores de remesas son relativamente más ricos que los hogares que no reciben remesas.

Lo anterior claramente nos muestra una etapa del proceso migratorio en la que los hogares que han migrado históricamente a los Estados Unidos con contratos de migración temporal, claramente superan en sus índices de riqueza a los hogares que no han participado en el proceso migratorio. El Cuadro 1 también permite observar que el porcentaje de hogares con menores a 5 años es de 20 por ciento en Caltimacán; es de 14 por ciento entre hogares receptores de remesas y es de 42 por ciento entre hogares no receptores de remesas, esta diferencia es significativa al 1 por ciento.

Por el contrario, existen diferentes variables en las que no se observan diferencias significativas. Dichas variables son el índice de aversión al riesgo, el índice de satisfacción ante la vida (Inegi, 2012), el número de integrantes promedio de un hogar, el porcentaje de hogares con niños mayores a 5 años, la edad del jefe del hogar, la edad de la madre, la educación del jefe del hogar, y la educación de la madre.

El Cuadro 2 nos muestra una posible explicación de por qué los gastos en educación en hogares receptores de remesas son mayores que entre hogares no receptores de remesas. La educación privada es más prevalente entre hogares receptores de remesas que entre hogares no receptores de remesas. Los datos muestran que los hogares que reciben remesas utilizan educación superior en los tres niveles de educación: básica, medio superior y superior. Los hogares que no reciben remesas solamente reportan un caso de educación media superior privada.

Cuadro 2. Número de estudiantes según nivel de escolaridad y tipo de escuela en Caltimacán, Hidalgo, 2016

\begin{tabular}{lcccc}
\hline $\begin{array}{c}\text { Nivel de } \\
\text { escolaridad }\end{array}$ & $\begin{array}{c}\text { Pública } \\
\text { No reciben } \\
\text { remesas }\end{array}$ & $\begin{array}{c}\text { Reciben } \\
\text { remesas }\end{array}$ & $\begin{array}{c}\text { Privada } \\
\text { No reciben } \\
\text { remesas }\end{array}$ & $\begin{array}{c}\text { Reciben } \\
\text { remesas }\end{array}$ \\
\hline Básico & 5 & 32 & 0 & 13 \\
Medio Superior & 0 & 18 & 1 & 6 \\
Superior & 1 & 8 & 0 & 8 \\
Total & 6 & 58 & 1 & 27 \\
\hline
\end{tabular}

Fuente: Elaboración propia con base en datos de la ERRICH-2016.

La encuesta preguntó únicamente los montos de remesas y su frecuencia de recepción en forma de intervalos, lo cual se presenta en el Cuadro 3. La mayoría de los hogares 
respondieron recibir remesas semanalmente, mientras que la segunda frecuencia muestra la recepción quincenal.

Para todos los grupos de frecuencia la moda en monto de recepción es más de 180 dólares, excepto para el grupo que recibe remesas quincenalmente para quienes la moda es recibir entre 105 y 170 dólares. Usando las frecuencias y montos recibidos, se estimó un promedio de remesas de 450 dólares mensuales. Estos montos de remesas están por debajo de los montos de remesas que estima BANXICO (2018). Para reducir el posible error de medición que tiene el monto de remesas, se utiliza la variable que define si los hogares reciben o no remesas.

Cuadro 3. Frecuencia y montos de recepción de remesas, Caltimacán, Hidalgo, 2016

\begin{tabular}{|c|c|c|c|c|}
\hline Montos/frecuencia & Semanal & Quincenal & Mensual & Bimestral \\
\hline 50 a 75 & $2 \%$ & $2 \%$ & 0 & 0 \\
\hline 80 a 100 & $5 \%$ & $5 \%$ & $22 \%$ & 0 \\
\hline 105 a 175 & $41 \%$ & $58 \%$ & $22 \%$ & 0 \\
\hline Más de 180 & $51 \%$ & $32 \%$ & $55 \%$ & $100 \%$ \\
\hline $\mathrm{N}$ & 39 & 34 & 9 & 1 \\
\hline
\end{tabular}

Fuente: Elaboración propia con base en datos de la ERRICH-2016.

\section{MODELO EMPÍRICO Y RESULTADOS}

En el modelo empírico, la variable dependiente es la proporción del gasto total que se destina a la educación y se plantea como una función de la recepción de remesas y de un conjunto de variables que buscan controlar por la capacidad de generar ingresos en el hogar, así como por otras características del hogar que puedan ayudar a determinar los gustos, preferencias y las necesidades de gasto en educación que existen en el mismo.

Existen variables que miden directamente el impacto de la inversión en educación tales como la permanencia escolar, la asistencia escolar o el rendimiento escolar de los miembros del hogar, entre otras. En este estudio, se mide la proporción de gasto en educación, lo cual se considera como una medida indirecta de la inversión del hogar en capital humano pues mide la intención de los hogares de aumentar sus conocimientos (Fermoso, 1997), y/o acumular más inversiones en educación (Bendfelt, 1992), y/o aumentar la productividad/calidad de sus hijos (Becker, Murphy y Tamura, 1990), y/o aumentar su 
calidad de vida (Bustamante, 2003; Martínez, 2006), en la medida en que la educación está asociada al nivel de bienestar subjetivo (Watanabe y Yasuko, 2005).

Esta estimación busca encontrar una ecuación de Engel, sin contar con información sobre precios y en la que se desea reducir la posible endogeneidad y causalidad inversa que puede generarse al incluir el ingreso (Adams y Cuecuecha, 2010). No se intenta estimar las proporciones marginales de ingreso que se propone estimar en Adams y Cuecuecha (2010) debido a que no se cuenta con el número de observaciones necesarias para establecer una estimación vía un sistema de ecuaciones. Específicamente, estimamos la siguiente ecuación:

$$
\begin{gathered}
\text { lped }_{i}=b_{0}+b_{1} \text { rem }_{i}+b_{2} \text { jefeduc }_{i}+b_{3} \text { indnocar }_{i}+b_{4} \text { mayor }_{i}+b_{5} \text { riesgo }_{i} \\
+b_{6} \text { actitud }_{i}+v_{i}
\end{gathered}
$$

Donde el logaritmo de la proporción del gasto a nivel del hogar es lped; rem es 1 si el hogar recibe remesas, 0 cualquier otro caso; la variable jefeduc es la escolaridad del jefe del hogar, y se incluye para medir la capacidad del hogar para generar ingreso (Becker, 1964), así como también para medir el posible conocimiento, actitudes y expectativas que los padres pueden tener para educar a sus hijos (Sawyer, 2015). La variable mayor 5 mide el número de hijos mayores a cinco años en la familia y que tienen menos de 14 años, se incluye para establecer si la familia tiene hijos en edad escolar. Se utiliza tanto para captar las necesidades que tiene la familia para gastar en educación como para captar las decisiones de cantidad (número de hijos) versus calidad (gastos en educación) que realiza el hogar (Becker, 1983).

La variable indnocar es un índice de no carencias que busca medir el bienestar objetivo de la familia, con el fin de medir la relación que el capital humano tiene con la calidad de vida (Becker, Murphy y Tamura, 1990; Bustamante, 2003; Martínez, 2006). Se consideró que al incluir una medición de no carencias en el hogar es posible medir parte del bienestar objetivo de las familias, para lo cual se siguieron los criterios para la medición multidimensional de la pobreza en México (Coneval, 2009).

La variable actitud mide la actitud ante la vida, la cual busca medir el grado de satisfacción de las familias, asociándola a los niveles de felicidad por los logros alcanzados en la vida, que están asociados al bienestar subjetivo (Watanabe y Yasuko, 2005) y que tiene relación con el estado de salud, situación económica, aspectos sociodemográficos y el progreso intergeneracional, tal como lo mide el Módulo de Bienestar Autorregulado, Bienestar subjetivo (Inegi, 2012). Por último, el modelo contempla la variable riesgo, que pretende medir la aversión al riesgo de las familias, el cual se sabe es un factor que puede afectar las decisiones de inversión de las familias. 
Es importante mencionar que el coeficiente de interés en esta estimación es el parámetro $b_{1}$, pues representa el efecto de las remesas sobre la inversión en educación. Dado que la ecuación de la proporción de gastos en educación se encuentra en logaritmos, los coeficientes de la ecuación pueden interpretarse como elasticidades. Esto será hecho a lo largo de la explicación de resultados.

La estimación de este parámetro enfrenta distintos retos; el primero es el número de observaciones de la muestra, lo que limita el número de variables a utilizar y hace no factible ciertas metodologías econométricas (Adams y Cuecuecha, 2010). El segundo se refiere a los estudios realizados sobre remesas con datos transversales encuentran, entre los que se encuentran (Adams, 2011):

1) la simultaneidad que existe entre la decisión de migrar con la decisión de enviar remesas, así como con otras decisiones como invertir en educación y fertilidad;

2) la auto selección de los emigrantes y receptores de remesas pues no todas las familias deciden enviar emigrantes, ni todas las familias reciben remesas;

3) la causalidad inversa que puede haber entre la decisión de invertir en educación y la recepción de las remesas, y

4) la presencia de sesgo de variables omitidas por la complejidad de las decisiones que los hogares toman. Entre las variables omitidas pueden estar las expectativas que los padres tienen sobre la educación de sus hijos, la inversión en gasto no monetario que los padres realizan en sus hijos, tales como tiempo de lectura o ayuda con las tareas escolares.

El tercer problema es el truncamiento ocasionado por el hecho de que los hogares que ya no tienen niños en edad escolar reportan gasto en educación igual a cero. Un cuarto problema es el potencial sesgo de medición, que tiene que ver con el hecho de que la encuesta, en su mayoría, fue contestada por las personas que se encontraban en el hogar, que su mayoría se trataba de mujeres, lo cual puede generar sesgo si ellas desconocen la información real. ${ }^{3}$

Para resolver los problemas de endogeneidad, posible causalidad inversa, variables omitidas, y de error de medición, las variables instrumento representan una solución en la medida en que encuentra una o más variables correlacionadas con la recepción de remesas, pero no con los no observables de la ecuación. Lograr lo anterior requiere de una estrategia empírica que en el caso de este artículo se presenta a continuación.

Tal como se explicó anteriormente, cada hogar en Caltimacán fue entrevistado preguntando específicamente por el lugar geográfico en el que trabajan sus familiares en los Estados Unidos de América. Con esta información se obtuvo el nivel de empleo y la tasa de creación de empleo en dicho estado de la Unión Americana para los años de 2010 a 2016.

${ }^{3}$ Los autores agradecemos a un árbitro anónimo por resaltar este potencial sesgo de medición. 
Para los casos de hogares sin migrantes, se calculó el promedio de creación de empleo en los 17 estados de la Unión Americana en la cual los caltimaquenses se encuentran trabajando, los cuales se especificaron en la primera sección de este artículo. Dado que lo anterior genera que los instrumentos sean iguales para dos observaciones: hogares que no cuentan familiares en los Estados Unidos, y hogares en los que los migrantes se localizan en el mismo estado de la Unión Americana, se multiplican las variables de empleo por el cuadrado de la edad del jefe del hogar. La información sobre el empleo se obtuvo del United States Department of Labor, Bureau of Labor Statistic (2016).

La existencia de una correlación estadísticamente significativa entre el empleo en el mercado laboral norteamericano y la recepción de remesas se explica por el hecho de que la actividad laboral en dicho mercado puede aumentar el número de caltimaquenses que se encuentra laborando en los Estados Unidos, las horas que ellos trabajan, así como el salario por hora lo que probablemente genera que los migrantes tengan más recursos para enviar en forma de remesas. Esta vinculación entre el envío de remesas a México y el mercado laboral de los Estados Unidos, parte de la relación existente entre el mercado laboral de los Estados Unidos y la emigración de Mexicanos a dicho país, la cual ha sido demostrada en la literatura (Rendón y Cuecuecha, 2010; Cuecuecha y Rendón, 2012).

Para lograr que el empleo en los Estados Unidos únicamente influencie la recepción de remesas, y no la ecuación de gasto en educación, se plantea seguir la estrategia de Adams y Cuecuecha $(2010,2013)$. Esta estrategia de identificación se basa en la estrategia de identificación que se utiliza para identificar modelos dinámicos de datos panel (Arellano y Bond, 1988).

Esta técnica se basa en asumir que el impacto del empleo en los Estados Unidos sobre la ecuación de gasto en educación solamente dura cuatro años, sin embargo, el impacto del empleo en Estados Unidos sobre la recepción de remesas dura al menos seis años. Para determinar el número de rezagos a ser incluidos en la ecuación, se siguió el principio de buscar el estadístico Cragg-Donald más alto, así como que la especificación respetara los tests estadísticos de Anderson y de Sargan (Adams y Cuecuecha, 2010).

El test de Cragg-Donald permite verificar que los instrumentos no son débiles, lo que implica que el sesgo generado por usar un instrumento es menor a la reducción en sesgo que se logra al resolver el problema de endogeneidad (Cragg y Donald, 1993). El estadístico de Anderson permite verificar que el modelo estimado no está subidentificado, es decir, que la matriz de instrumentos es de rango mayor al número de ecuaciones que se desea estimar, en nuestro caso, una ecuación. El estadístico J de Hansen permite determinar si los rezagos incluidos como instrumentos de sobra, de hecho son ortogonales a los residuales de la ecuación de gasto. Las pruebas estadísticas y la estimación con instrumentos lineales se realizaron utilizando una rutina desarrollada por Baum, Schaffer y Stillman (2007). 
Al escoger la combinación de instrumentos que obtiene el Cragg-Donald más alto, se garantiza que se utiliza el subespacio de instrumentos que mayor información genera relativa al sesgo de estimación implícito al usar instrumentos, garantizando que dicho subespacio conforma un conjunto de instrumentos válido y que identifica la ecuación de gasto (Cragg y Donald, 1993).

El modelo a estimar con variables instrumento se presenta en la ecuación (2):

$$
\begin{aligned}
\text { lped }_{i}=b_{0}+ & b_{1} \text { rem }_{i}+b_{2} \text { jefeduc }_{i}+b_{3} \text { indnocar }_{i}+b_{4} \text { mayor }_{i}+b_{5} \text { riesgo }_{i} \\
& +b_{6} \text { actitud }_{i}+b_{7} \text { empE }_{2013}+b_{8} \text { empE }_{2014}+b_{9} \text { empE }_{2015} \\
& +b_{10} \text { empEU }_{2016}+\sigma E\left[v_{i} \mid \text { lped }_{i}>0\right]+u_{i}
\end{aligned}
$$

La ecuación (2) incluye el elemento $\sigma E\left[v_{i} \mid \operatorname{lped}_{i}>0\right]$ que corrige por el truncamiento de la ecuación (1). De igual forma, se incluyen las variables empE $U_{2016}$ a $e m p E U_{2013}$, las cuales están asociadas a la tasa de creación de empleo en los Estados Unidos para los años 2013 a 2016. Se introdujeron bajo el argumento de que al condicionar por sus valores, las tasas de creación de empleo en EU en los años 2010, el cuadrado de la creación de empleo en 2010 y el cuadrado de la creación de empleo en 2011 funcionan como instrumentos para la recepción de remesas en el hogar.

En estimaciones exploratorias realizadas por los autores, se introdujo el nivel de Empleo en EU en 2012. Sin embargo, el estadístico Cragg-Donald reducía su valor y por lo mismo se decidió excluir a dicha variable tanto del conjunto de instrumentos como del conjunto de variables de control. El supuesto explícito mencionado anteriormente se ilustra en la ecuación (3):

$$
\operatorname{cov}\left(\operatorname{emp} E U_{2010}, v_{2016} \mid \operatorname{emp} E U_{2016}, \ldots, \operatorname{emp} E U_{2013}, x\right)=0
$$

Un supuesto similar se hace para las otras dos variables instrumento. Esta ecuación no incluye información macroeconómica para México debido a que todas las observaciones pertenecen a una misma localidad y por lo tanto todas ellas están sujetas a los mismos shocks nacionales. Por el contrario, el hecho de que cada hogar tenga familiares en distintos puntos de la Unión Americana, implica que cada uno de los hogares está expuesto a diferentes shocks regionales en los Estados Unidos. La ecuación que se utiliza para estimar la recepción de remesas es entonces la siguiente:

$$
\begin{aligned}
& \text { rem }_{i}=\alpha_{0}+\alpha_{1} \text { jefeduc }_{i}+\alpha_{2} \text { indnocar }_{i}+\alpha_{3} \text { mayor }_{i}+\alpha_{4} \text { riesgo }_{i}+\alpha_{5} \text { actitud }_{i} \\
& +\alpha_{6} e m p E U_{2010}+\alpha_{7} e m p E U_{2010}{ }^{2}+\alpha_{8} e m p E U_{2011}{ }^{2}+\alpha_{9} e m p E U_{2013} \\
& +\alpha_{10} e m p E U_{2014}+\alpha_{11} e m p E U_{2015}+\alpha_{12} e m p E U_{2016}+\varepsilon_{i}
\end{aligned}
$$


Donde los rezagos incluidos, así como la decisión de incluirlos lineales o cuadráticos, se tomó siguiendo el principio de lograr el mayor estadístico Cragg-Donald.

Para enfrentar el problema de la posible simultaneidad en la educación del jefe del hogar, se tomó la decisión de hacer estimaciones en las que dicha variable es sustituida por la educación de la madre, así como estimaciones en las que la educación del jefe del hogar es excluida de la estimación. De igual forma, se decidió estimar especificaciones excluyendo la variable relacionada con la presencia de niños mayores a cinco años (mayor5) debido a que se encontró en los modelos no lineales (Tobit) que dicha variable reduce la validez de los instrumentos, lo cual es evidencia de que la presencia de niños mayores a 5 años es una variable muy probablemente endógena.

Finalmente, se hicieron estimaciones en las que se incluyó el logaritmo del gasto del hogar. Esto permite identificar si la proporción de gasto en educación se modifica proporcionalmente con el ingreso -lo cual ocurre en funciones de gasto derivadas de funciones de demanda con homogeneidad de grado uno en ingreso-, o si la proporción de gasto varía no proporcional al ingreso, situación que puede derivarse si al aumentar el ingreso los individuos gastan proporcionalmente más en educación (la educación es un bien de lujo), o bien si al aumentar el ingreso los gastos en educación crecen menos que proporcionalmente ante el ingreso (la educación es un bien inelástico al ingreso).

\section{RESULTADOS}

El Cuadro 4 muestra los resultados de estimar una versión simplificada de la ecuación 1, así como diferentes versiones de la ecuación 2, que se plantean una relación entre el logaritmo de la fracción del gasto en educación y la recepción de las remesas, controlando por la educación, la aversión al riesgo y la satisfacción ante la vida. La columna 1 del Cuadro 4 presenta el modelo de mínimos cuadrados ordinarios (MCO) incluyendo únicamente la variable remesas en el hogar. Se encuentra una asociación positiva entre las remesas y la fracción de gasto en educación. En particular, el resultado implica que los hogares que reciben remesas tienen una fracción de gasto 0.81 por ciento mayor a la fracción de gasto de los hogares sin remesas.

La columna 2 del Cuadro 4, presenta la misma estimación usando el modelo Tobit, que controla por el hecho de no todos los hogares presentan gastos en educación. La asociación positiva se mantiene y se estima que los hogares que reciben remesas tienen una proporción de gasto en educación 0.30 por ciento mayor a la de los hogares sin remesas. La columna 3 del Cuadro 4 incluye en la estimación a las variables de aversión al riesgo y de posición ante la vida, obteniendo resultados cualitativamente similares. Esto implica que el efecto encontrado no se explica por elementos de aversión al riesgo o de bienestar subjetivo. 
Cuadro 4. Estimaciones para el logaritmo del gasto en educación,

Caltimacán Hidalgo, 2016

\begin{tabular}{|c|c|c|c|c|c|}
\hline Variable & $\mathrm{MCO}$ & Tobit & Tobit & Tobit & Tobit \\
\hline Remesas en el hogar & $\begin{array}{l}0.810 * \\
{[0.437]}\end{array}$ & $\begin{array}{c}0.299 * * \\
{[0.136]}\end{array}$ & $\begin{array}{l}0.272 * * \\
{[0.140]}\end{array}$ & $\begin{array}{c}0.315 * * \\
{[0.147]}\end{array}$ & $\begin{array}{l}0.251 * \\
{[0.148]}\end{array}$ \\
\hline $\begin{array}{l}\text { Educación del jefe del } \\
\text { hogar }\end{array}$ & & & & $\begin{array}{c}0.029 * * \\
{[0.014]}\end{array}$ & \\
\hline Educación de la madre & & & & & $\begin{array}{l}0.020 * * \\
{[0.011]}\end{array}$ \\
\hline Aversión al riesgo & & & $\begin{array}{l}-0.157 \\
{[0.110]}\end{array}$ & $\begin{array}{l}-0.130 \\
{[0.115]}\end{array}$ & $\begin{array}{l}-0.172 \\
{[0.118]}\end{array}$ \\
\hline $\begin{array}{l}\text { Satisfacción ante la } \\
\text { vida }\end{array}$ & & & $\begin{array}{l}-0.026 \\
{[0.110]}\end{array}$ & $\begin{array}{l}-0.025 \\
{[0.114]}\end{array}$ & $\begin{array}{l}-0.038 \\
{[0.119]}\end{array}$ \\
\hline Constante & $\begin{array}{l}-2.336 * * * \\
{[0.423]}\end{array}$ & NA & NA & NA & NA \\
\hline $\mathrm{N}$ & 63 & 102 & 102 & 102 & 102 \\
\hline $\mathrm{R}^{2}$ ajustada & $8.9 \%$ & NA & NA & NA & NA \\
\hline Pseudo $\mathrm{R}^{2}$ & NA & $1.73 \%$ & $2.9 \%$ & $3.7 \%$ & $3.7 \%$ \\
\hline
\end{tabular}

Notas: Errores Estándar entre corchetes. ***Significativos al 1\%. **Significativos al 5\%. Significativos al 10\%. NA: No aplica.

Fuente: Elaboración propia con base en datos de la ERRICH-2016.

De igual forma las columnas 4 y 5 incluyen en la estimación la educación del jefe del hogar y la educación de la madre, sin que se alteren cualitativamente los resultados, pues se obtiene una asociación positiva entre las remesas y la fracción de gasto en la educación, con un efecto marginal entre 0.25 por ciento y 0.32 por ciento. En la columna 4 del Cuadro 4 puede verse que la educación del padre resulta ser estadísticamente significativa, e implica que un aumento de 1 por ciento en la educación del padre genera un aumento de 0.03 por ciento en la fracción de gasto en la educación.

En la columna 5 del Cuadro 4 se observa que la educación de la madre es estadísticamente significativa, e implica que un incremento de 1 por ciento en la educación de la madre aumenta 0.02 por ciento la fracción de gasto en educación. En todos los casos mostrados en el Cuadro 4, la varianza explicada por los modelos es pequeña, pues varía entre 1.7 por ciento y 8.9 por ciento.

El Cuadro 5 presenta estimaciones del modelo en su versión Tobit, incluyendo una por una las variables que en el análisis se encontró pueden explicar la asociación observada entre las remesas y la fracción de gasto en educación. La columna 1 del Cuadro 5 incluye, en relación a la columna 5 del Cuadro 4, el índice de no carencias. Al incluir dicha variable, todas las variables se hacen no significativas, pero el grado de varianza explicada se 
mantiene en los niveles reportados en el Cuadro 4. Esto sugiere que el índice de no carencias tiene una alta correlación con el resto de las variables incluidas en la regresión.

En la columna 2 del Cuadro 5 se añade, en relación a la quinta columna del Cuadro 4, únicamente el logaritmo del gasto. Esto también genera que la asociación entre las remesas y la fracción de gasto en el hogar se haga no significativa. El gasto en el hogar es significativo a 1 por ciento e implica que un crecimiento de 1 por ciento en el gasto del hogar genera un aumento de 0.56 por ciento en la fracción de gasto en educación.

En la columna 3 del Cuadro 5 se incluye, en relación a la columna 5 del Cuadro 4, la variable de niños mayores de 5 años. El resultado es que también se pierde la asociación entre las remesas y la fracción de gasto en educación. Los hogares que tienen niños mayores a 5 años tienen una fracción de gasto en educación 0.56 por ciento mayor a la de los hogares sin niños mayores a 5 años. En ambas columnas se logra explicar alrededor de 10 por ciento de la varianza observada.

La columna 4 del Cuadro 5 presenta la estimación con todas las variables presentadas en la ecuación 3. Se mantiene el resultado de que no hay asociación entre las remesas y la fracción de gasto en el hogar y se mantiene la significancia estadística del logaritmo del gasto y de la variable niños mayores a cinco años.

Estos resultados tienen varias implicaciones. La primera es que existen restricciones de acceso al crédito en Caltimacán, Hidalgo, que se expresan por el hecho de que tener niños en edad escolar obliga a las familias a gastar más en educación, y que las remesas ayudan a disminuir estas restricciones crediticias, permitiendo a los hogares de Caltimacán pagar sus gastos en educación, tal como lo prevé la Teoría de la Nueva Economía de la Migración Laboral (Stark y Bloom, 1985).

Este resultado se comprueba, pues la inclusión en la estimación del ingreso del hogar y de una variable asociada a la riqueza también explican la asociación observada entre las remesas y la fracción de gasto en la educación. Una segunda implicación es que el acceso al crédito muy probablemente está asociado al gasto total de los hogares, como lo comprueba que en presencia de la variable de gasto no se observa un coeficiente significativo para la variable índice de no carencias. Este resultado probablemente se explica por el hecho de que en Caltimacán existen pocos accesos a servicios financieros formales, por lo que muy probablemente el crédito existente disponible para la población opera a través de mecanismos informales basados en la observación de los niveles de gasto de los hogares de Caltimacán.

Cuadro 5. Efectos marginales para el logaritmo del gasto en educación, modelos Tobit

Con Con

Variable riqueza riqueza,




\begin{tabular}{|c|c|c|c|c|}
\hline & & $\begin{array}{c}\text { Con } \\
\text { logaritmo } \\
\text { del gasto } \\
\end{array}$ & $\begin{array}{c}\text { Con } \\
\text { fertilidad }\end{array}$ & $\begin{array}{l}\text { gasto y } \\
\text { fertilidad }\end{array}$ \\
\hline Remesas en el hogar & $\begin{array}{c}0.206 \\
{[0.144]}\end{array}$ & $\begin{array}{c}-0.032 \\
{[0.171]}\end{array}$ & $\begin{array}{c}0.238 \\
{[0.149]}\end{array}$ & $\begin{array}{c}-0.123 \\
{[0.178]}\end{array}$ \\
\hline Niños mayores de 5 años & & & $\begin{array}{c}0.560 * * * \\
{[0.107]}\end{array}$ & $\begin{array}{c}0.616 * * * \\
{[0.109]}\end{array}$ \\
\hline Gasto total en el hogar & & $\begin{array}{c}0.487 * * * \\
{[0.115]}\end{array}$ & & $\begin{array}{c}0.544 * * * \\
{[0.157]}\end{array}$ \\
\hline $\begin{array}{l}\text { Índice de no carencias } \\
\text { Aversión al riesgo y Satisfacción } \\
\text { ante la vida }\end{array}$ & Sí & No & No & Sí \\
\hline Pseudo $\mathrm{R}^{2}$ & $2.7 \%$ & $8.6 \%$ & $10.7 \%$ & $17.6 \%$ \\
\hline
\end{tabular}

***Significativo al 1\%. **Significativo al 5\%.*Significativo al $10 \%$.

Fuente: Cálculos propios con base en la ERRICHH-2016.

Es igualmente importante destacar que el resultado contrasta con los encontrados por Adams y Cuecuecha (2010), en los que se muestra que en Guatemala hay un impacto positivo de las remesas sobre la fracción de gasto en educación. Este resultado puede estar asociado a la naturaleza de la migración de Caltimacán, en la que muchos de los emigrantes utilizan contratos temporales de trabajo que usan continuamente desde hace ya muchos años, lo cual les permite ver los aumentos en el ingreso de las familias como permanentes.

De igual modo, los contratos temporales de trabajo les permite mantener un contacto permanente con la sociedad en Caltimacán; de tal modo que sus patrones de gasto simplemente se ajustan al nivel de ingreso que tienen y observacionalmente mantienen patrones de gasto similares a hogares que no reciben remesas y que tienen niveles de ingreso similares a ellos. Esto explicaría por qué desde un punto de vista teórico, las remesas son fungibles con el resto de ingresos en Caltimacán, Hidalgo.

En el Cuadro 6 se presenta la estimación de la ecuación de las remesas (ecuación 4) bajo dos versiones y dos tipos de modelos. Primero se presenta la estimación usando modelos lineales y modelos Tobit. Se presenta una versión de la ecuación 4, en la cual se excluyen de la estimación las variables de logaritmo del gasto, índice de no carencias y niños mayores de 5 años. En la segunda versión, para el modelo lineal se incluyen todas las variables, excepto la de niños mayores a 5 años, lo cual se hace en virtud de que los análisis de validez de instrumentos para el modelo Tobit rechazan el que la variable de niños mayores a 5 años pueda incluirse en la ecuación. Por consistencia, en el modelo lineal se decidió presentar la estimación excluyendo dicha variable. Es importante mencionar que esto demuestra que en Caltimacán los niños mayores a 5 años están ya en edad de riesgo para migrar a los Estados Unidos, al punto que su presencia en Caltimacán está asociada a los movimientos del mercado laboral en los Estados Unidos. 
La primera columna del Cuadro 6 excluye las variables de educación, riqueza y gasto. Dicha columna nos muestra que todos los instrumentos -Empleo en EUA en 2010, el cuadrado del Empleo en EUA en 2010 y el cuadrado del Empleo en EUA en 2011- son estadísticamente significativos al 1 por ciento. Las variables de empleo que nos permiten controlar por la correlación contemporánea entre la fracción de gasto en educación y el empleo en EUA -Empleo en EUA en 2013, Empleo en EUA en 2014, Empleo en EUA en 2015 y Empleo en EUA en 2016- son estadísticamente significativas al 1 por ciento, con excepción del Empleo en Estados Unidos en 2016 que no es estadísticamente significativa. El modelo de primera etapa puede explicar un 73 por ciento de la variación observada en la recepción de remesas en Caltimacán.

El anterior resultado es muy importante pues en análisis preliminares se encontró que hay una alta correlación entre las variables de empleo en los EUA, arriba de 0.9 por ciento. Este dato en sí mismo no prueba la multicolinealidad, pues en modelos multivariados la correlación simple deja de ser informativa (Klein, 1962). El problema de multicolinealidad menos que perfecta se caracteriza por generar una matriz de varianzas covarianzas con un valor de condición alto, así como por generar una estimación de varianza sesgada (Maddala, 1996). Esto genera una $R^{2}$ alta pero muchos coeficientes $t$ de Student pequeños.

Sin embargo, es importante mencionar que se utilizó el paquete Stata 15 el cual se caracteriza por usar algoritmos que son robustos a la multicolinealidad. El hecho de que se obtenga en la estimación parámetros altamente significativas y una $\mathrm{R}^{2}$ alta demuestra que las estimaciones no son afectadas por el problema de multicolinealidad menos que perfecta. ${ }^{4}$

El Cuadro 6 también nos muestra que los instrumentos identifican la ecuación de remesas debido a que el test de Anderson se rechaza al 1 por ciento; también nos muestran que los instrumentos que sobre identifican la ecuación de remesas son exógenos en relación a los errores de la ecuación de fracción de gasto en educación debido a que el test J de Hansen no rechaza la hipótesis de exogeneidad de instrumentos; finalmente también nos muestran que los instrumentos no son débiles debido a que el test de Crag-Donald indica que los coeficientes tienen un sesgo máximo relativo de 5 por ciento y un sesgo máximo de 10 por ciento (Stock y Yogo, 2005). La segunda columna del Cuadro 6 muestra los resultados de la ecuación 4 al incluir las variables de educación del jefe del hogar, la educación de la madre, el logaritmo del gasto y el índice de no carencias.

Los instrumentos son todos estadísticamente significativos y el modelo logra explicar una variación de 76 por ciento en la ecuación de remesas, el test de Anderson rechaza que la ecuación de remesas esté subidentificada, el test J de Hansen muestra que la ecuación está

\footnotetext{
${ }^{4}$ Se agradece a un evaluador anónimo que nos alertó sobre la necesidad de evaluar la posible presencia de multicolinealidad. Los resultados sobre correlaciones entre las variables de empleo se encuentran disponibles y pueden solicitarse a los autores.
} 
sobreidentificada, y el test de Crag-Donald muestra que hay un sesgo máximo relativo de 5 por ciento. El único cambio con relación a la columna 1 del mismo cuadro es que el test de Crag-Donald está por debajo del valor que nos indica un sesgo máximo de 10 por ciento. Esto implica que esta ecuación puede presentar un sesgo máximo ligeramente mayor al 10 por ciento.

La tercera columna muestra los resultados de estimar el modelo usando un Tobit y los instrumentos, así como excluyendo la educación, el logaritmo del gasto y la riqueza. Todas las variables de Empleo en EUA son significativas a 1 por ciento, excepto la de Empleo en EUA en 2016, la cual es significativa al 10 por ciento. Para este modelo no lineal, los tests que es apropiado utilizar (Finlay y Magnusson, 2009) son el logaritmo de verosimilitud condicional (CLR, por sus siglas en inglés), el estadístico de Anderson-Rubin, el Multiplicador de Lagrange (LM, por sus siglas en inglés) y el estadístico LM-J. En todos los casos se prueba la hipótesis nula de que el sesgo generado por el uso de instrumentos es cero. La columna 3 del Cuadro 6 nos muestra que en todos los casos no se rechaza la hipótesis nula por lo que se comprueba la fortaleza de los instrumentos. La cuarta columna del Cuadro 6 muestra la estimación de la ecuación 4, incluyendo todas las variables del modelo. Todos los resultados son cualitativamente similares a los mencionados en la tercera columna del mismo cuadro.

Cuadro 6. Resultados de primera etapa, recepción de remesas en hogares

\begin{tabular}{|c|c|c|c|c|}
\hline Variable & $\begin{array}{l}\text { Excluye } \\
\text { educación, } \\
\text { riqueza y } \\
\text { gasto }\end{array}$ & $\begin{array}{c}\text { Con } \\
\text { educación, } \\
\text { riqueza y } \\
\text { gasto }\end{array}$ & $\begin{array}{l}\text { Excluye } \\
\text { educación, } \\
\text { riqueza y } \\
\text { gasto }\end{array}$ & $\begin{array}{c}\text { Con } \\
\text { educación, } \\
\text { riqueza y } \\
\text { gasto }\end{array}$ \\
\hline $\begin{array}{l}\text { Aversión al riesgo y } \\
\text { satisfacción ante la vida }\end{array}$ & Sí & Sí & Sí & Sí \\
\hline $\begin{array}{l}\text { Empleo generado en EUA en } \\
2010\end{array}$ & $\begin{array}{c}2.90 \mathrm{E}- \\
06 * * * \\
{[1.12 \mathrm{E}-06]}\end{array}$ & $\begin{array}{c}3.25 \mathrm{E}-06 * * \\
{[1.37 \mathrm{E}-06]}\end{array}$ & $\begin{array}{c}4.65 \mathrm{E}- \\
06 * * * \\
{[8.20 \mathrm{E}-07]}\end{array}$ & $\begin{array}{c}4.81 \mathrm{E}- \\
06 * * * \\
{[9.59 \mathrm{E}-07]}\end{array}$ \\
\hline $\begin{array}{l}\text { Empleo generado en EUA en } \\
2010^{\wedge} 2\end{array}$ & $\begin{array}{c}-1.51 \mathrm{E}- \\
12 * * * \\
{[2.63 \mathrm{E}-13]}\end{array}$ & $\begin{array}{l}-1.45 \mathrm{E}- \\
12 * * * \\
{[2.86 \mathrm{E}-13]}\end{array}$ & $\begin{array}{l}-1.59 \mathrm{E}- \\
12 * * * \\
{[1.42 \mathrm{E}-13]}\end{array}$ & $\begin{array}{l}-1.53 \mathrm{E}- \\
12 * * * \\
{[1.61 \mathrm{E}-13]}\end{array}$ \\
\hline $\begin{array}{l}\text { Empleo generado en EUA en } \\
2011^{\wedge} 2\end{array}$ & $\begin{array}{c}1.48 \mathrm{E}- \\
12 * * * \\
{[2.55 \mathrm{E}-13]}\end{array}$ & $\begin{array}{l}1.41 \mathrm{E}- \\
12 * * * \\
{[2.78 \mathrm{E}-13]}\end{array}$ & $\begin{array}{l}1.56 \mathrm{E}- \\
12 * * * \\
{[1.38 \mathrm{E}-13]}\end{array}$ & $\begin{array}{l}1.50 \mathrm{E}- \\
12 * * * \\
{[1.57 \mathrm{E}-13]}\end{array}$ \\
\hline $\begin{array}{l}\text { Empleo generado en EUA en } \\
2013\end{array}$ & $\begin{array}{c}1.68 \mathrm{E}- \\
05 * * * \\
{[5.12 \mathrm{E}-06]}\end{array}$ & $\begin{array}{l}1.45 \mathrm{E}-05 * * \\
{[5.56 \mathrm{E}-06]}\end{array}$ & $\begin{array}{l}1.17 \mathrm{E}- \\
05 * * * \\
{[2.80 \mathrm{E}-06]}\end{array}$ & $\begin{array}{l}1.03 \mathrm{E}- \\
05 * * * \\
{[3.04 \mathrm{E}-06]}\end{array}$ \\
\hline $\begin{array}{l}\text { Empleo generado en EUA en } \\
2014\end{array}$ & $\begin{array}{c}-4.33 \mathrm{E}- \\
05 * * * \\
{[1.06 \mathrm{E}-05]}\end{array}$ & $\begin{array}{l}-3.97 \mathrm{E}- \\
05 * * * \\
{[1.18 \mathrm{E}-05]}\end{array}$ & $\begin{array}{l}-3.89 \mathrm{E}- \\
05 * * * \\
{[6.24 \mathrm{E}-06]}\end{array}$ & $\begin{array}{l}-3.68 \mathrm{E}- \\
05 * * * \\
{[6.71 \mathrm{E}-06]}\end{array}$ \\
\hline $\begin{array}{l}\text { Empleo generado en EUA en } \\
2015\end{array}$ & $\begin{array}{l}2.54 \mathrm{E}- \\
05 * * *\end{array}$ & $\begin{array}{l}2.37 \mathrm{E}- \\
05^{* * *}\end{array}$ & $\begin{array}{l}2.46 \mathrm{E}- \\
05^{* * *}\end{array}$ & $\begin{array}{l}2.40 \mathrm{E}- \\
05 * * *\end{array}$ \\
\hline
\end{tabular}




\begin{tabular}{|c|c|c|c|c|}
\hline $\begin{array}{l}\text { Empleo generado en EUA en } \\
2016\end{array}$ & $\begin{array}{l}-6.81 \mathrm{E}-07 \\
{[4.78 \mathrm{E}-07]}\end{array}$ & $\begin{array}{l}-6.27 \mathrm{E}-07 \\
{[6.25 \mathrm{E}-07]}\end{array}$ & $\begin{array}{l}-7.56 \mathrm{E}-07 * \\
{[4.12 \mathrm{E}-07]}\end{array}$ & $\begin{array}{l}-8.25 \mathrm{E}-07 * \\
{[4.66 \mathrm{E}-07]}\end{array}$ \\
\hline Constante & $\begin{array}{c}1.167 * * * \\
{[0.110]}\end{array}$ & $\begin{array}{l}0.487 \\
{[0.718]}\end{array}$ & $\begin{array}{l}1.298 * * * \\
{[0.106]}\end{array}$ & $\begin{array}{l}0.875^{*} \\
{[0.469]}\end{array}$ \\
\hline Modelo & Lineal & Lineal & Tobit & Tobit \\
\hline $\mathrm{R}^{2}$ centrada & $73.7 \%$ & $76.5 \%$ & NA & NA \\
\hline Anderson $\mathrm{Chi}^{2}(3)$ & $81.2 * * *$ & $52.1 * * *$ & NA & NA \\
\hline Crag-Donald F test & $46.4^{\mathrm{a}}$ & $21.01^{\mathrm{a}}$ & NA & NA \\
\hline Hansen J Chi ${ }^{2}(2)$ & 0.71 & 0.37 & NA & NA \\
\hline $\begin{array}{l}\text { Logaritmo de verosimilitud } \\
\text { condicional (CLR) }\end{array}$ & NA & NA & 0.17 & 1.43 \\
\hline Anderson-Rubin & NA & NA & 0.17 & 1.43 \\
\hline $\begin{array}{l}\text { Multiplicador de verosimilitud } \\
\text { (LM) }\end{array}$ & NA & NA & 0.17 & 1.43 \\
\hline Estadístico LM-J & NA & NA & NR 5\% & NR 5\% \\
\hline
\end{tabular}

El Cuadro 7 muestra los resultados para la ecuación del logaritmo de la fracción del gasto en educación, es decir, los resultados instrumentados. La primera columna del Cuadro 7 presenta los resultados del modelo lineal. Las remesas son estadísticamente significativas y los hogares receptores de remesas tienen una fracción de gasto en educación 1.04 por ciento mayor a la de los hogares sin remesas. Este coeficiente es mayor al encontrado en el modelo lineal y en el modelo Tobit. El modelo puede explicar 14 por ciento de la variación observada en los datos.

La segunda columna del Cuadro 7 muestra la estimación de la ecuación 4 incluyendo todas las variables. El impacto de las remesas desaparece y se encuentra que la educación de la madre y el gasto en el hogar son los únicos estadísticamente significativos. Un aumento de 1 por ciento en la educación de la madre genera un aumento del 0.05 por ciento en la fracción de gasto en educación. Un aumento de 1 por ciento en el gasto del hogar, aumenta 0.60 por ciento la fracción de gasto en educación.

La tercera columna del Cuadro 7 muestra la estimación excluyendo educación, riqueza y gasto. Nuevamente, se obtiene que los hogares que reciben remesas tienen una fracción de gasto en educación que es 0.36 por ciento mayor a la de los hogares sin remesas. Este parámetro es menor al estimado usando modelos lineales, pero mayor al estimado sin instrumentos. Esto implica que el truncamiento en los datos genera un sesgo al alza en el parámetro estimado, mientras que la no instrumentación genera un sesgo a la baja en el parámetro estimado. El modelo es estadísticamente significativo al 10 por ciento. 
Cuadro 7. Modelos lineales (coeficientes) y modelos Tobit (efectos marginales) para el gasto en educación

\begin{tabular}{|c|c|c|c|c|}
\hline Variable & $\begin{array}{l}\text { Excluye } \\
\text { educación, } \\
\text { riqueza y } \\
\text { gasto }\end{array}$ & $\begin{array}{c}\text { Con } \\
\text { educación, } \\
\text { riqueza y } \\
\text { gasto }\end{array}$ & $\begin{array}{l}\text { Excluye } \\
\text { educación, } \\
\text { riqueza y } \\
\text { gasto }\end{array}$ & $\begin{array}{c}\text { Con } \\
\text { educación, } \\
\text { riqueza y } \\
\text { gasto }\end{array}$ \\
\hline Remesas en el hogar & $\begin{array}{l}1.040 * * \\
{[0.453]}\end{array}$ & $\begin{array}{c}0.313 \\
{[0.525]}\end{array}$ & $\begin{array}{c}0.364 * * * \\
{[0.137]}\end{array}$ & $\begin{array}{c}0.049 \\
{[0.230]}\end{array}$ \\
\hline Educación del padre & & $\begin{array}{c}-0.030 \\
{[0.032]}\end{array}$ & & $\begin{array}{c}-0.002 \\
{[0.020]}\end{array}$ \\
\hline Educación de la madre & & $\begin{array}{l}0.046^{*} \\
{[0.028]}\end{array}$ & & $\begin{array}{c}0.022 \\
{[0.015]}\end{array}$ \\
\hline Gasto total en el hogar & & $\begin{array}{l}0.602 * * \\
{[0.290]}\end{array}$ & & $\begin{array}{c}0.473 * * * \\
{[0.121]}\end{array}$ \\
\hline Incluye índice de carencias & No & Sí & No & Sí \\
\hline $\begin{array}{l}\text { Incluye aversión al riesgo y } \\
\text { satisfacción ante la vida }\end{array}$ & Sí & Sí & Sí & Sí \\
\hline $\begin{array}{l}\text { Empleo generado en EUA de } \\
2013 \text { a } 2016\end{array}$ & Sí & Sí & Sí & Sí \\
\hline Constante & $\begin{array}{c}-3.014 * * \\
{[0.543]}\end{array}$ & $\begin{array}{c}-7.609 * * * \\
{[2.311]}\end{array}$ & NA & NA \\
\hline Modelo & Lineal & Lineal & Tobit & Tobit \\
\hline $\mathrm{R}^{2}$ centrada & $13.8 \%$ & $25.7 \%$ & NA & NA \\
\hline Wald Chi ${ }^{2}$ & NA & NA & $12.3^{*}$ & $42.9 * * *$ \\
\hline $\mathrm{N}$ & 63 & 63 & 100 & 100 \\
\hline
\end{tabular}

***Significativo al 1\%. $* *$ Significativo al $5 \%$.

Fuente: Cálculos propios con base en la ERRICHH-2016.

La cuarta columna del Cuadro 7 muestra la estimación de la ecuación 4 sin incluir la variable de niños mayores de 5 años. Nuevamente, el impacto de las remesas desaparece y solamente es estadísticamente significativo el gasto del hogar. En particular, se encuentra que un aumento del 1 por ciento en el gasto de los hogares, genera un aumento del 0.47 por ciento en la fracción de gasto en educación. El modelo es significativo al 1 por ciento. Estos resultados confirman que el efecto de las remesas está asociado a la existencia de restricciones crediticias, mismas que las remesas ayudan a reducir (Stark y Bloom, 1985). De igual forma, muestran que los hogares de Caltimacán que reciben remesas ajustan sus patrones de gasto a niveles similares a los de hogares con ingresos similares a los de ellos, independientemente de si los ingresos provienen de las remesas o no.

\section{CONCLUSIONES}

Los resultados muestran que los hogares que reciben remesas tienen una proporción de mayor gasto en educación; sin embargo, lo anterior es explicado por la mayor existencia de 
niños mayores de 5 años en los hogares receptores de remesas, lo cual nos muestra que existen restricciones crediticias en la comunidad de estudio, pues el tener niños en edad obligatoria de ir a la escuela puede explicar las diferencias en gasto entre hogares con remesas y sin remesas. Además, se encuentra que el efecto de las remesas también puede ser explicado por el índice de no carencias, es decir, la riqueza de las familias, así como por el logaritmo del gasto de los hogares, es decir, por el nivel de ingreso de los hogares.

Lo anterior confirma que existen restricciones crediticias en Caltimacán, Hidalgo, y permite ver que el acceso al crédito está vinculado a los niveles de ingreso, y no a los niveles de riqueza, pues al introducir juntas las variables de riqueza y de gasto en el hogar, solamente la segunda mantiene su significancia estadística.

Estos resultados implican que la recepción de remesas funciona como estrategia para evitar las restricciones crediticias (Stark y Bloom, 1985), y que probablemente pueden generar problemas de desigualdad en la comunidad, pues la recepción de remesas aumenta los niveles de ingreso y de acceso al crédito en la comunidad. De igual manera, los resultados también dan crédito a la hipótesis de que la proporción de gasto en educación varía en proporción al ingreso, por lo que las remesas, más allá de aumentar los ingresos, no tienen ya un efecto adicional, lo que demostraría que los ingresos de los hogares son fungibles .

Este resultado puede deberse a la naturaleza de la migración de la comunidad de estudio, primordialmente legal vía contratos temporales, así como a la madurez que tiene la migración en la localidad, pues ya casi todos los habitantes tienen familiares en Estados Unidos. Estos hechos generan que los caltimaquenses asuman sus aumentos de ingresos como permanentes, y simplemente ajusten sus niveles de gasto en educación de acuerdo a sus niveles de ingreso. Este resultado contrasta por lo encontrado en otros estudios (Adams y Cuecuecha, 2010; Sawyer, 2015) y confirma lo encontrado por Kandell y Massey (2002) acerca de la importancia del contexto particular a estudiar.

Este estudio tiene otras importantes implicaciones que se derivan de ser un estudio a nivel localidad. La primera es la identificación de los lugares en los que trabajan los migrantes en los Estados Unidos, lo que permitió construir instrumentos con una variación a nivel de hogar y no a nivel comunidad como en otros estudios (Adams y Cuecuecha, 2010). La segunda es mostrar la relevancia de las características específicas de la migración de la localidad de estudio para entender los efectos de las remesas. La tercera es mostrar la importancia de estudios específicos a nivel localidad con un número suficiente de observaciones para realizar estimaciones cuantitativas. Una limitante del presente estudio es que no es una base de datos longitudinal, lo que sería recomendable dada la naturaleza dinámica del proceso migratorio, y de las inversiones en educación.

Por último, es importante mencionar que el tener un mejor conocimiento sobre el esfuerzo que realiza la población emigrante de Caltimacán al emigrar y mantener la inversión en 
educación, permite reconocer el aporte de los emigrantes en la mejora del capital humano, el bienestar y la calidad de vida en su lugar de origen.

\section{REFERENCIAS}

Acosta Rangel, R. E. y Caamal Olvera, C. (2017). Las remesas y la permanencia escolar en México. Migraciones Internacionales, 9(2), 85-111.

Adams, R. (2011). Evaluating the Economic Impact of International Remittances on Developing Countries Using Household Surveys: A Literature Review. Journal of Development Studies 47(6), 809-828.

Adams, R. y Cuecuecha, A. (2010). Remittances, Household Expenditure and Investment in Guatemala. World Development, 38(11), 1626-1641.

Adams, R. y Cuecuecha, A. (2013). The Impact of Remittances on Investment and Poverty in Ghana. World Development, (50), 24-40.

Alaminos, A., López Fernández, C., López Monzalve, B., Perea Crespo, I. y Santacreu, O. (2009). El retorno de las migraciones circulares: La regulación de las migraciones profesionales. Revista Obets, (3), 59-70.

Arellano, M. y Bond, S. (1988). Dynamic panel data estimation using DPD. The Institute for Fiscal Studies. Working Paper Series, 88-15.

Banco de México (BANXICO). (2018). Balanza de Pagos: Remesas. Sistema de Información Económica. Consultado en junio 2018. Disponible en: http://www.banxico.org.mx/SieInternet/consultarDirectorioInternetAction.do?sector=1 \&accion $=$ consultarCuadroAnalitico $\&$ idCuadro $=\mathrm{CA} 11 \&$ locale $=\mathrm{es}$

Baum, C. F., Schaffer, M. E. y Stillman, S. (2007). IVREG2: Stata module for extended instrumental variables/2SLS and GMM estimation. Disponible en: http://ideas.repec.org/c/boc/bocode/s425401.html

Becker, G. S., Murphy, K. M. y Tamura, R. (1990). Human capital, fertility and economic growth. Journal of Political Economy, 90 (5), 12-37.

Becker, G. S. (1983). El capital humano. Un análisis teórico y empírico referido fundamentalmente a la educación. España, Madrid: Editorial Alianza.

Becker, G. S. (1964) Human Capital. Nueva York: Columbia University Press for the National Bureau of Economic Research. Recuperado de: http://econpapers.repec.org/bookchap/nbrnberbk/beck75-1.htm

Bendfelt J. F. (1992). La dimensión desconocida del capital: El capital humano. En J.F. Bendfelt (Ed.) La Educación en Crisis (pp. 27-74). Guatemala: Centro de Estudios Económicos Sociales.

Bryant, J., Giorguli Saucedo, S. y Hernández Padilla, E. (2016). International Migration and Academic Performance of Mexican Adolescents. International Migration Review, 50(4), 1-38. 
Bustamante, N. (2003). Educación y pobreza en la ciudad de Medellín: Evidencia Empírica. Revista Ruido Blanco, (1), 35-47.

Buvinic, M. y Gupta, G. R. (1997). Female-Headed Households and Female-Maintained Families: Are They Worth Targeting to Reduce Poverty in Developing Countries? Economic Development and Cultural Change, 45(2), 259-280.

Consejo Nacional de Población (Conapo) (2010). Índices de intensidad migratoria, MéxicoEstados Unidos 2010. Documento electrónico. Disponible en http://www.conapo.gob.mx/swb/CONAPO/Indices_de_intensidad_migratoria_Mexico -Estados_Unidos_2010

Consejo Nacional de Evaluación (Coneval) (2009). Metodología para la medición multidimensional de la pobreza en México. México. Disponible en http://www.coneval.org.mx/Informes/Coordinacion/INFORMES_Y_PUBLICACION ES_PDF/Metodologia_Multidimensional_web.pdf

Cox Edwards, A. y Ureta, M. (2003). International Migration, Remittances and Schooling: Evidence from El Salvador. Journal of Development Economics 72, 429-461. Recuperado de: http://www.uh.edu/ adkugler/Cox\&Ureta.pdf

Cragg, J. G. y Donald, S. G. (1993). Testing Identifiability and Specification in Instrumental Variables Models. Econometric Theory, 9, 222-240.

Cuecuecha, Alfredo y Adams, R. (2016). The economic impact of international remittances on poverty and household consumption and investment in Indonesia. Journal of Finance and Economics, 4(3), 12-31.

Cuecuecha, A. y Rendón, S. (2012). Mexicans In and Out of the US: Facts on Job Search and International Migration. En A. Cuecuecha y C. Pederzini (Eds.) Migration and remittances from Mexico: trends, impacts and new challenges (pp. 119-142). Lanham: Lexington Books.

Deaton, A. (1997). The Analysis of Household Surveys: A microeconometric approach to development policy. Washington: World Bank.

Durand, J. (2016). Historia mínima de la migración México-Estados Unidos. México: Colegio de México.

Fermoso, P. (1997). Manual de economía de la educación. Madrid: Narcea.

Finlay, K. y Magnusson, L. M. (2009). Implementing weak-instrument robust tests for a general class of instrumental-variables models. The Stata Journal, 9(3), 398-421.

Fundación BBVA Bancomer A.C. (2017). Anuario de Migración y Remesas 2017. Disponible en: https://www.bbvaresearch.com/wpcontent/uploads/2017/08/1707 AnuarioMigracionRemesas 2017.pdf

García Nájera, Y. (2017). Uso de remesas internacionales en la inversión en educación, en la comunidad de Caltimacán, Hidalgo. (Tesis Doctoral). El Colegio de Tlaxcala A. C. Tlaxcala, San Pablo Apetatitlán. 
Hanson, G. y Woodruff, C. (2003). Emigration and Educational Attainment in Mexico. Mimeo. University of California, San Diego Working Paper: last updated, 1-38.

Hanson, G., Robertson, R. y Spilimbergo, A. (2002). Does border enforcement protect U.S. workers from illegal immigration? The Review of Economics and Statistics, 84(1), 7392.

Instituto Nacional de Estadística y Geografía (Inegi). (2012). Metodología. Módulo de Bienestar Autorreportado. Disponible en: https://www.inegi.org.mx/investigacion/bienestar/ampliado/default.html\#Documentaci on

Inegi. (2010). Censo de Población y Vivienda. Documentos conceptuales y metodológicos. INEGI. México. Disponible en: https://www.inegi.org.mx/app/biblioteca/ficha.html?upc=702825002065

Kandell, W. y Massey, D. S. (2002). The Culture of Mexican Migration: A Theoretical and Empirical Analysis. Social Forces, 80(3), 981-1004.

Klein, L. R. (1962). An Introduction to Econometrics. Englewood Cliffs: Prentice Hall.

Levitt, P. (1998). Social Remittances: Migration Driven Local-Level Forms of Cultural Difusion. International Migration Review, 32(4), 926-948.

López Córdova, E. (2005). Globalization, Migration and Development: The Role of Mexican Migrant Remittances. Economía, 6(1), 1-39.

Maddala, G. S. (1996). Introducción a la Econometría. México: Prentice Hall.

Martínez, J. (2006). Notas sobre migración y desarrollo local, una mirada desde las remesas de los migrantes. Santiago de Chile: CEPAL/CELADE.

Massey, D. (2016). The Mexico-US Border in the American Imagination. Proceedings of the American Philosophical Society, 160(2), 160-177.

McKenzie, D. y Rapoport, H. (2006). Can Migration Reduce Educational Attainments? Depressing Evidence from Mexico? Policy Research Working Paper, 1, 1-42. Recuperado de http://documents.worldbank.org/curated/en/929791468045585109/Can-migrationreduce-educational-attainment-Evidence-from-Mexico

Pérez Gañán, M. R. y Pesántez Calle, B. N. (2017). El impacto migratorio en las aspiraciones y expectativas educativas y de movilidad social en jóvenes sigseños. Migraciones Internacionales, 9(2), 57-84.

Quezada Ramírez, M. F. (2008). La migración hñäñu del Valle del Mezquital, estado de Hidalgo. México: Comisión Nacional para el Desarrollo de los pueblos indígenas..

Ray, D. (1998). Development Economics. Nueva York: Princeton University Press.

Rendón, S. y Cuecuecha, A. (2010). International Job Search: Mexicans in and out of the US. Review of Economics of the Household, 8(1), 53-82.

Sawyer, A. (2015). Migración, remesas y escolarización: ¿Estímulos o amenazas para la Educación para Todos en México? Revista Latinoamericana de Educación Comparada, 6(8), 76-90. 
Stark, O. y Bloom, D. E. (1985). The New Economics of Labor Migration. American Economic Review, 75(2), 173-178.

Stock, J. y Yogo, M. (2005). Testing for weak instruments in linear IV regression. En D. W. $\mathrm{K}$. Andrews y J. Stock (Eds.). Identification and Inference for Econometric Models, (pp. 80-108). Nueva York: Cambridge University Press.

United States Departament of Labor, Bureau of Labor Statistic (2016). Recuperado de: https://www.bls.gov/

Valdivieso Taborga, C. E., Valdivieso Castellón, R. y Valdivieso Taborga, O. A. (2011). Determinación del tamaño muestral mediante el uso de árboles de decisión. Revista Investigación \& Desarrollo, (11), 48-176.

Watanabe, A. y Yasuko, B. (2005). La capacidad y el bienestar subjetivo como dimensiones de estudio de la calidad de vida. Revista Colombiana de Psicología, (14), 73-79.

World Bank Group. (2018). Migration and Remittances: Recent Developments and OutlookTransit Migration. Migration and Development Brief, (29), 1-51. Washington, DC: World Bank. Recuperado de:

https://openknowledge.worldbank.org/handle/10986/29777

Wooldridge, J. (2013). Introduction to Econometrics. London: Cengage Learning.

Yang, D. (2008). International Migration, Human Capital and Entrepreneurship: Evidence from Philippine Migrants' Exchange Rate Shocks. The Economic Journal, (118), 591630. 